Pacific Journal of Mathematics

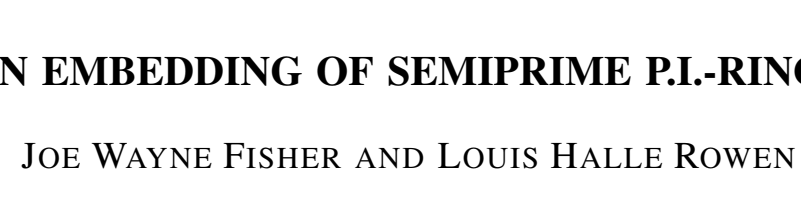




\title{
AN EMBEDDING OF SEMIPRIME P.I.-RINGS
}

\author{
Joe W. Fisher and Louis Halle Rowen
}

Let us say an extension $R^{\prime}$ of a ring $R$ is a quotient ring of $R$ if every regular element of $R$ is invertible in $R^{\prime}$. In this note we construct a class of quotient rings of semiprime $P . I$.-rings and use this construction to find rapid proofs of several facts about semiprime P.I.-rings.

1. Preliminaries. Throughout this paper $R$ will denote a semiprime P.I.-ring with unity and center $C$, i.e., $R$ has no nonzero nilpotent ideals and the standard polynomial

$$
S_{2 n}\left(X_{1}, \cdots, X_{2 n}\right)=\Sigma_{\pi}(\operatorname{sgn} \pi) X_{\pi(1)} \cdots X_{\pi(2 n)},
$$

the sum taken over all permutations $\pi$ of $(1, \cdots, 2 n)$, is an identity of $R$ for suitable $n$ (the minimal such $n$ is the degree of $R$ ). Formanek [5] has constructed a polynomial $g_{n}\left(X_{1}, \cdots, X_{n+1}\right)$ which is central for all semiprime P.I.-rings of degree $n$, and Rowen [11] has used these central polynomials to prove

TheOREM A. Any nonzero ideal of $R$ intersects $C$ nontrivially.

Let $S=\{c \in C: c r \neq 0$ for all nonzero $r$ in $R\}$. Define an equivalence relation on $R \times S$ by saying $\left(r_{1}, s_{1}\right) \sim\left(r_{2}, s_{2}\right)$ if $r_{1} s_{z}=r_{2} s_{1}$, and let $r s^{-1}$ denote the equivalence class of $(r, s)$. Then $R_{S}=\left\{r s^{-1}\right.$ : $(r, s) \in R \times S\}$ is a ring when endowed with the (well-defined) operations $r_{1} s_{1}^{-1}+r_{2} s_{2}^{-1}=\left(r_{1} s_{2}+r_{2} s_{1}\right)\left(s_{1} s_{2}\right)^{-1}$, called the ring of central quotients of $R$. The following theorem is a direct consequence of Theorem A (cf., Rowen [11, §2]):

Theorem B. If $R$ is a prime P.I.-ring of degree $n$, then $R_{S}$ is simple Artinian of dimension $n^{2}$ over its center $C_{S}, C_{S}$ is the quotient field of $C$, and $R_{S}$ satisfies the identities of $R$.

Theorem $\mathrm{B}$ often enables us to study $R$ by examining $R_{S}$. If $R$ is a semiprime P.I.-ring of degree $n$ and satisfies the ascending chain condition on annihilators of two-sided ideals, then $R_{S}$ is the classical semisimple Artinian ring of left and right quotients of $R$ (cf., [12]). Unfortunately, this situation fails for semiprime P.I.rings in general, so one is led to study other extensions of $R$. The purpose of this paper is to introduce a straightforward type of extension of $R$ and to deduce from it properties of semiprime P.I.rings and their classical quotient rings (if these exist). This paper 
subsumes Fisher [4]. First we shall derive some easy known properties of $R$.

For a subset $A$ of $R$, let $\operatorname{Ann}_{R}(A)$ denote $\{r \in R \mid A r=0\}$. Also we say an ideal $A$ of $R$ is essential if for every nonzero ideal $B$ of $R, A \cap B \neq 0$. Since $R$ is semiprime, $A \cap B=0$ if and only if $A B=$ 0 . The following lemma is known by Martindale [9].

Lemma 1. (i) If $E$ is an essential ideal of $C$, then $E R$ is an essential ideal of $R$.

(ii) If $J$ is a left ideal of $R$ with $\operatorname{Ann}_{R}(J)=0$, then $J \cap C$ is essential in $C$, so $J$ contains an essential ideal of $R$.

Proof. (i) Suppose that $A \cap E=0$ for some ideal $A$ of $R$. Then $(A \cap C) \cap E=A \cap(C \cap E)=A \cap E=0$, implying $A \cap C=0$. Hence $A=0$ by Theorem $\mathrm{A}$ and thus $E R$ is essential.

(ii) Viewed as a ring (without 1 ), $J$ is clearly a P.I.-ring and can easily be shown to be semiprime. We claim that $J \cap C=$ cent $J$. Indeed $J \cap C \subseteq$ cent $J$ and if $a \in$ cent $J$, then for all $r$ in $R$ and for all $x$ in $J,(r a-a r) x=r a x-a(r x)=r a x-r(x a)=r a x-r a x=0$. Hence $(r a-a r) \in \operatorname{Ann}_{R}(J)=0$ and so $a \in C$.

Now let $B$ be an ideal of $C$ such that $(J \cap C) \cap B=0$. Then $(J \cap C \cap B R)^{2} \subseteq(J \cap C) B R=B(J \cap C) R \subseteq(B \cap(J \cap C)) R=0$ and so $(J \cap C \cap B R)^{2}=0$. Since $J \cap C$ has no nonzero nilpotent elements, we have $J \cap C \cap B R=0$, i.e., $(J \cap C) \cap(J \cap B R)=0$. But by Theorem A applied to the semiprime ring $J$ (with center $J \cap C$ ), $J \cap B R=0$. This implies $R J B=B R J \subseteq J \cap B R=0$, so $B \subseteq \operatorname{Ann}_{R}(R J)=$ Ann $J=$ 0 . Hence $J \cap C$ is essential in $C$. The rest of the lemma follows from (i).

2. Definition and elementary properties of $T(R)$. For the remainder of this paper, we assume that the semiprime P.I.-ring $R$ has degree $n$. This implies that every prime factor ring of $R$ has degree equal to or less than $n$. The degree of a prime ideal $P$ of $R$ is defined as the degree of $R / P$.

Let $\mathscr{P}$ be a collection (indexed by $\Lambda$ ) of prime ideals $P_{\lambda}$ of $R$ such that $\bigcap\left\{P_{\lambda}: \lambda \in \Lambda\right\}=0$. For each $\lambda$ in $\Lambda$, set $R_{\lambda}=R / P_{\lambda}$, let $Q_{\lambda}$ equal the simple Artinian ring of central quotients of $R_{\lambda}$, and let $Q$ be the complete direct product $\Pi\left\{Q_{\lambda}: \lambda \in \Lambda\right\}$. There is a natural embedding $R \rightarrow \Pi R_{\lambda} \rightarrow Q$ and we shall often view $R$ as a subring of $Q$ under this embedding. Hence $R$ satisfies the identities of $Q$. On the other hand, any identity $f$ of $R$ is an identity of each $R_{\lambda}$, and is an identity of each $Q_{\lambda}$ by Theorem $\mathrm{B}$; hence $f$ is an identity of $Q=\Pi Q_{\lambda}$. Consequently, $R$ and $Q$ satisfy the same identities. 
Clearly $Q$ is von Neumann regular, i.e., for any $x \in Q$, there is some $y$ in $Q$ such that $x y x=x$.

As remarked above, each $Q_{\lambda}$ has degree $\leqq n$. Let $\Lambda_{j}=\left\{\lambda \in \Lambda_{i}: Q_{\lambda}\right.$ has degree $j\}$ and let $\bar{Q}_{j}=\Pi\left\{Q_{\lambda}: \lambda \in \Lambda_{j}\right\}$. Then $\bar{Q}_{j}$ is a semiprimitive ring of degree $j$ with the property that every nonzero homomorphic image of $\bar{Q}_{j}$ has degree $j$. This is equivalent to saying, by the Artin [2]-Procesi [10] theorem, that $\bar{Q}_{j}$ is an Azumaya algebra of rank $j$. Hence $Q$ is a finite direct sum of the Azumaya algebras $\bar{Q}_{j}$ of finite rank $j$.

Lemma 2. Any nonzero homomorphic image $\psi(Q)$ of $Q$ is von Neumann regular. Moreover, $\psi(Q)$ is the finite direct sum of the Azumaya algebras $\psi\left(\bar{Q}_{j}\right)$ of finite rank $j$, and each identity of $R$ is an identity of $\psi(Q)$.

Proof. Every homomorphic image of a von Neumann ring is von Neumann regular. Also, every homomorphic image of $\psi\left(\bar{Q}_{j}\right)$ is a homomorphic image of $\bar{Q}_{j}$, thereby having rank $j$; hence $\psi\left(\bar{Q}_{j}\right)$ is Azumaya of rank $j$, and clearly $\psi(Q)$ is the direct sum of $\psi\left(\bar{Q}_{j}\right)$ for $j=1, \cdots, n$. The last assertion is immediate.

For any $x$ in $Q$, let $x_{\lambda}$ denote the component of $x$ in $Q_{\lambda}$ and let $W_{x}=\left\{\lambda \in \Lambda: x_{\lambda} \neq 0\right\}$. Set $V=\left\{x \in Q: \bigcap\left\{P_{\lambda}: \lambda \in W_{x}\right\}\right.$ is an essential ideal of $R$ \}. Now $V$ is an ideal of $Q$ because, taking $x, y$ in $V$ and $q$ in $Q, W_{x \pm y} \subseteq W_{x} \cup W_{y} ; W_{q x} \subseteq W_{x} ; W_{x q} \leqq W_{x}$. Let us define $T(R, \mathscr{P})=$ $Q / V$. From Lemma 2 we have that $T(R, \mathscr{P})$ is a finite direct sum of Azumaya algebras of finite rank and is von Neumann regular.

Theorem 1. (i) There is a canonical imbedding $R \rightarrow T(R, \mathscr{P})$ given by $R \rightarrow Q \rightarrow Q / V$.

(ii) Half regular elements of $R$ are both left and right invertible in $T(R, \mathscr{P})$.

(iii) $T(R, \mathscr{P})$ satisfies precisely the same identities as $R$.

Proof. (i) We need show only that $R \cap V=0$. If $r \in R \cap V$, then $\bigcap\left\{P_{\lambda}: \lambda \in W_{r}\right\}$ is essential in $R$ and so $\bigcap\left\{P_{\lambda}: r \in P_{\lambda}\right\}=0$. Hence $r=0$.

(ii) Let $r$ in $R$ have right annihilator zero. Then $\operatorname{Ann}_{R}(R r)=$ 0 and $R r$ contains an essential ideal $E$ of $C$ by Lemma 1(ii). Let $W_{r}^{\prime}=\left\{\lambda: P_{\lambda} \nsupseteq E\right\}$. Clearly $W_{r}^{\prime} \subseteq W_{r}$. Moreover, for any $\lambda$ in $W_{r}^{\prime}$ there is an $x_{\lambda}$ in $Q_{\lambda}$ such that $0 \neq x_{\lambda} r_{\lambda} \in$ cent $Q_{\lambda}$. Since cent $Q_{\lambda}$ is a field, there is $d_{\lambda}$ in cent $Q_{\lambda}$ such that $d_{\lambda} x_{\lambda} r_{\lambda}=1_{\lambda}$. Furthermore, $r_{\lambda} d_{\lambda} x_{\lambda}=1_{\lambda}$ because $Q_{\lambda}$ is simple Artinian. Define $y$ in $Q$ as follows: $y_{\lambda}=0$ for $\lambda \notin W_{r}^{\prime}$ and $y_{\lambda}=d_{\lambda} x_{\lambda}$ for $\lambda \in W_{r}^{\prime}$. Then $(y r-1)_{\lambda}=0$ and $(r y-1)_{\lambda}=0$ for all $\lambda$ in $W_{r}^{\prime}$. Thus $\bigcap\left\{P_{\lambda}: \lambda \in W_{y r-1}\right\} \supseteqq \bigcap\left\{P_{\lambda}: \lambda \notin W_{r}^{\prime}\right\} \supseteqq$ 
$E$. It follows from Lemma 1 (i) that $y r-1 \in V$; likewise $r y-1 \in V$. Hence, for $\bar{y}$ the image of $y$ in $T(R, \mathscr{P})$, we have $\bar{y} r=1$ and $r \bar{y}=$ 1 in $T(R, \mathscr{P})$.

(iii) $T(R, \mathscr{P})$ satisfies each identity of $R$ by Lemma 2; conversely, by (i), each identity of $T(R, \mathscr{P})$ is an identity of $R$.

The following theorem of Herstein-Small [8] is a consequence of Theorem 1.

COROLLARY 1. Half regular elements of $R$ are regular.

Proof. If $r$ in $R$ is, say, right regular, then for some $y \in T(R$, $\mathscr{P})$ we have $r y=1$. Hence $r$ is left regular.

Corollary 2. If $R$ has a classical left ring of quotients $R^{\prime}$, then $R^{\prime}$ satisfies the same polynomial identities as $R$.

Proof. In view of Theorem 1(ii) the canonical embedding of $R$ into $T(R, \mathscr{P})$ extends to an embedding of $R^{\prime}$ into $T(R, \mathscr{P})$. Hence $R^{\prime}$ satisfies the identities of $T(R, \mathscr{P})$ which are precisely the identities of $R$.

Note that this construction of $T(R, \mathscr{P})$ is related to constructions of Amitsur [1] and Goldie [7]. Also, those versed in logic may wish to regard $T(R, \mathscr{P})$ as the "reduced product" (cf., [6]) of the simple Artinian rings $\left\{Q_{\lambda}: \lambda \in \Lambda\right\}$ by the filter $\left\{\Lambda-W_{x}: x \in V\right\}$.

3. Definition and structure of $T(R)$. Now we consider an interesting special case of $T(R, \mathscr{P})$. Index the set of all the prime ideals of $R$ by a set $\bar{\Lambda}$ with $\bar{\Lambda}_{i}=\left\{\lambda \in \bar{\Lambda}: P_{\lambda}\right.$ has degree $\left.i\right\}$ for $i=1$, $\cdots, n$. Set $\bar{N}_{i}=\bigcap\left\{P_{\lambda}: \lambda \in \bar{\Lambda}_{i}\right\}$ (if $\bar{\Lambda}_{i}=\phi$ then $\left.\bar{N}_{i}=R\right), \Lambda_{i}=\left\{\lambda \in \bar{\Lambda}_{i}\right.$ : $\left.P_{\lambda} \nsupseteq \bigcap_{j=i+1}^{n} \bar{N}_{j}\right\}, \mathscr{P}_{i}=\left\{P_{\lambda}: \lambda \in \Lambda_{i}\right\}, \mathscr{P}=\mathscr{P}_{1} \cup \cdots \cup \mathscr{P}_{n}, \Lambda=\Lambda_{1} \cup \cdots \cup \Lambda_{n}$. Clearly $\cap\{P: P \in \mathscr{P})=\bar{N}_{1} \cap \cdots \cap \bar{N}_{n}=0$. We define $T(R)$ to be $T(R, \mathscr{P})$. Note that $\Lambda_{n}=\bar{\Lambda}_{n}$ and that $\Lambda=\Lambda_{n}$ if and only if $\bar{N}_{n}=0$.

Let $N_{i}=\bigcap\left\{P: P \in \mathscr{F}_{i}\right\}$ and let $R_{i}=R / N_{i}$. Note that $N_{n}=\bar{N}_{n}$. Clearly $R$ is a subdirect product of the $R_{i}$ and this subdirect decomposition is unique with respect to the properties that each of the nonzero subdirect factors has a degree different from each of the other subdirect factors and that for any subdirect factor of degree $j$, the intersection of its prime ideals of degree $j$ is zero. Our aim is to show how the structure of $T(R)$ is linked to this decomposition. As in Rowen [12], let a polynomial be called regular if it is linear in some indeterminant, and let the central kernel of a ring be the additive subgroup generated by the values taken (in the center) by regular central polynomials of the ring. The central kernel is an ideal of the center $C$. If the central kernel is essential in $C$, we 
say that $R$ has essential central kernel. Let $I$ be the central of $R$, let $B=N_{1} \cap \cdots \cap N_{n-1}$, and let $R_{n}^{\prime}=R / B$. It is shown in Rowen [12] that for $\lambda \in \bar{\Lambda}, I \nsubseteq P_{\lambda}$ if and only if $\lambda \in \Lambda_{n}$.

Lemma 3. (i) $\left(R I+N_{n}\right) / N_{n}$ is an essential ideal of $R_{n}$.

(ii) $\left(N_{n}+B\right) / B$ is an essential ideal of $R_{n}^{\prime}$.

(iii) A semiprime ring $R$ of degree $j$ has essential central kernel if and only if the intersection of its prime ideals of degree $j$ is zero.

Proof. (i) Suppose that $\left[\left(A+N_{n}\right) / N_{n}\right] \cap\left[\left(R I+N_{n}\right) / N_{n}\right]=0$ for some ideal $A$ of $R$. Then $A R I \subseteq N_{n} \subseteq P_{\lambda}$ for each $\lambda \in \Lambda_{n}$. Since $I \nsubseteq P_{\lambda}$ for $\lambda \in \Lambda_{n}$, we have $A \subseteq \bigcap\left\{P_{\lambda}: \lambda \in \Lambda_{n}\right\}=N_{n}$. So

$$
\left(A+N_{n}\right) / N_{n}=0 .
$$

(ii) Suppose that $[(A+B) / B] \cap\left[\left(N_{n}+B\right) / B\right]=0$ for some ideal $A$ of $R$. Then $A N_{n} \leqq B=N_{1} \cap \cdots \cap N_{n-1} \leqq P_{\lambda}$ for each $\lambda \in \Lambda-\Lambda_{n}$. By definition $P_{\lambda} \nsupseteq N_{n}$ for $\lambda \in \Lambda-\Lambda_{n}$, so $A \subseteq \bigcap\left\{P_{\lambda}: \lambda \in \Lambda-\Lambda_{n}\right\}=B$. So $(A+B) / B=0$.

(iii) Let $\bar{N}_{j}$ be the intersection of the prime ideals of degree $j$. Since every prime ideal of degree $<j$ contains $I$, we have $I \cap \bar{N}_{j}=0$. Since $I$ is essential in $C$, we have $\bar{N}_{j} \cap C=0$, hence $N_{j}=0$ by Theorem A. The reverse implication is immediate from (i) and Lemma 1.

Lemma 3(iii) gives us a neater characterization of $R_{1}, \cdots, R_{n}$. Namely, the nonzero $R_{i}$ are uniquely determined if we are to express $R$ as a subdirect product of minimal length of rings with essential central kernel.

Lemma 4. (i) Suppose that $J$ is an ideal of $R$ and $N_{n} \subseteq J$. Then $J$ is essential in $R$ if and only if $J / N_{n}$ is essential in $R_{n}$.

(ii) Suppose $B \subseteq J$. Then $J$ is essential in $R$ if and only if $J / B$ is essential in $R_{n}^{\prime}$.

Proof. (i) $(\Longrightarrow)$ Suppose that $J / N_{n} \cap\left[\left(A+N_{n}\right) / N_{n}\right]=0$ for some ideal $A$ of $R$. Then $J A \subseteq N_{n}$ and so $B \cap J A=0$. Now since $I \subseteq$ $P_{\lambda}$ for each $\lambda \in \Lambda-\Lambda_{n}$, we have $R I \subseteq \bigcap\left\{P_{\lambda}: \lambda \in \Lambda-\Lambda_{n}\right\} \subseteq B$ and $R I \cap J A=0$, or $I J A=0$. Hence $(J \cap A I)^{2} \leqq(J A I)^{2}=0$ and $J \cap A I=$ 0 since $R$ is semiprime. By hypothesis, we then see $A I=0$, so $A \subseteq N_{n}$ by Lemma 3(i). Consequently $\left(A+N_{n}\right) / N_{n}=0$.

Conversely suppose that $J \cap A=0$ for some ideal $A$ of $R$. Then $J A=0 \subseteq N_{n}$, so $A \subseteq N_{n}$ by hypothesis. Thus $A^{2} \subseteq N_{n} A \subseteq J A=0$ and so $A=0$.

(ii) $(\Rightarrow)$ Suppose that $J / B \cap[(A+B) / B]=0$. Then $J A \subseteq B$, or 
$J A N_{n} \subseteq B \cap N_{n}=0$ which implies $A N_{n}=0$. Hence $A \subseteq B$ by Lemma 3(ii) and so $(A+B) / B=0$. The proof of the converse is analogous to that in (i).

THEOREM 2. $T(R) \cong T\left(R_{1}\right) \oplus \cdots \oplus T\left(R_{n}\right)$.

Proof. We use induction on $n=$ degree of $R$. The assertion is true for $n=2$. Since $R_{n}^{\prime}$ has degree $\leqq n-1$, we have by our induction hypothesis that $T\left(R_{n}^{\prime}\right) \cong T\left(R_{1}\right) \oplus \cdots \oplus T\left(R_{n-1}\right)$. Let $\bar{Q}_{n}=$ $\Pi\left\{Q_{i}: \lambda \in \Lambda_{n}\right\}, \bar{Q}_{n}^{\prime}=\Pi\left\{Q_{\lambda}: \lambda \in \Lambda-\Lambda_{n}\right\}, V_{n}=V \cap \bar{Q}_{n}$, and $V_{n}^{\prime}=V_{n} \cap \bar{Q}_{n}^{\prime}$. Clearly $V=V_{n} \oplus V_{n}^{\prime}$ and $T(R)=Q / V \cong \bar{Q}_{n} \oplus \bar{Q}_{n}^{\prime} / V \cong \bar{Q}_{n}^{\prime} / V_{n} \oplus \bar{Q}_{n}^{\prime} / V_{n}^{\prime}$. But Lemma 4(i) shows $\bar{Q}_{n} / V_{n} \cong T\left(R_{n}\right)$ and Lemma 4(ii) shows $\bar{Q}_{n}^{\prime} / V_{n}^{\prime} \cong$ $T\left(R_{n}^{\prime}\right)$. Thus $T(R) \cong T\left(R_{n}\right) \oplus T\left(R_{n}^{\prime}\right) \cong T\left(R_{1}\right) \oplus \cdots \oplus T\left(R_{n-1}\right) \oplus T\left(R_{n}\right)$.

Theorem 2 enables us to reduce the study of $T(R)$ to rings with essential central kernel.

THEOREM 3. Let $R$ be a semiprime P.I.-ring of degree $n$ with essential central kernel. Then $T(R)$ is an Azumaya algebra of rank $n^{2}$ and $T(C) \cong$ center $(T(R))$.

Proof. By Lemma 3(iii), $N_{n}=0$. Hence $T(R)$ is a homomorphic image of $\Pi\left\{Q_{\lambda}: \lambda \in \Lambda_{n}\right\}$. Therefore, $T(R)$ is Azumaya of rank $n^{2}$. Write $C_{\lambda}=$ center $Q_{\lambda}$ for $\lambda \in \Lambda$. Since $\Pi\left\{Q_{\lambda}: \lambda \in \Lambda_{n}\right\}$ is an Azumaya algebra of rank $n^{2}$, we have the following fact which we will need later, cent $\left[\left(\Pi_{\lambda \in A_{n}} Q_{\lambda}\right) /\left(V \cap \Pi_{\lambda \in A_{n}} Q_{\lambda}\right)\right]=\left(\Pi_{\lambda \in A_{n}} C_{\lambda}+V \cap \Pi_{\lambda \in A_{n}} Q_{\lambda}\right) /(V \cap$ $\left.\Pi_{\lambda \in I_{n}} Q_{i}\right)$.

We claim that the homomorphism $\varphi:\left(\Pi_{\lambda \in \Lambda} Q_{\lambda}\right) / V \rightarrow\left(\Pi_{\lambda \in A_{n}} Q_{\lambda}\right) /(V \cap$ $\left.\Pi_{\lambda \in \lambda_{n}} Q_{\lambda}\right)$, induced by the projection, $\Pi_{\lambda \in \Lambda} Q_{\lambda} \rightarrow \Pi_{\lambda \in \Lambda_{n}} Q_{\lambda}$, is an isomorphism. Indeed, suppose that $0 \neq x+V$ for $x$ in $\Pi_{\lambda \in \Lambda} Q_{\lambda}$. Then $\bigcap\left\{P_{\lambda}: \lambda \in W_{x}\right\}$ is not essential. Since each prime of degree $<n$ contains $I$ and $I \cong \bigcap\left\{P_{\lambda}: \lambda \in W_{x} \cap\left(\Lambda-\Lambda_{n}\right)\right\}$ is essential, we conclude that $\bigcap\left\{P_{\lambda}: \lambda \in\right.$ $\left.W_{x} \cap A_{n}\right\}$ is not essential and $0 \neq x+\left(V \cap \Pi_{\lambda \in A_{n}} Q_{\lambda}\right)$. Consequently $\varphi$ is an isomorphism.

Now by Rowen [12, Theorem 3] there exists a 1:1 correspondence of $\left\{P_{\lambda}: \lambda \in \Lambda_{n}\right\}$ and the set of prime ideals of $C$, not containing $I$, given by $P_{\lambda} \rightarrow P_{\lambda} \cap C$. We claim that $T(C) \cong\left(\Pi_{\lambda \in A_{n}} C_{\lambda}\right) /\left(V \cap \Pi_{\lambda \in A_{n}} C_{\lambda}\right)$. The proof of this is similar to the one in the preceding paragraph because every prime in $C$ which is not in $\left\{P_{\lambda} \cap C: \lambda \in \Lambda_{n}\right\}$ contains $I$ which is essential in $C$.

Finally we have all the requisite pieces to obtain 


$$
\begin{aligned}
T(C) & \cong\left(\Pi_{\lambda \in \Lambda_{n}} C_{\lambda}\right) /\left(V \cap \Pi_{\lambda \in A_{n}} C_{\lambda}\right) \\
& \cong\left(\Pi_{\lambda \in A_{n}} C_{\lambda}+V \cap \Pi_{\lambda \in \Lambda_{n}} Q_{\lambda}\right) /\left(V \cap \Pi_{\lambda \in A_{n}} Q_{\lambda}\right) \\
& \cong \operatorname{cent}\left[\left(\Pi_{\lambda \in \Lambda_{n}} Q_{\lambda}\right) /\left(V \cap \Pi_{\lambda \in \Lambda_{n}} Q_{\lambda}\right)\right] \\
& \cong \operatorname{cent}\left(\left(\Pi_{\lambda \in \Lambda} Q_{\lambda}\right) / V\right)=\operatorname{cent}(T(R)) .
\end{aligned}
$$

REMARK 1. Given $\mathscr{P}$ as in $\S 2$, let $\varphi: Q \rightarrow T(R, \mathscr{P})$ be the canonical homomorphism. Then there is a partial order on fideals $A$ of $Q: \operatorname{Ker} \varphi \subseteq A$ and $R \cap A=0\}$. So there exists a maximal such ideal $\bar{A}$. Then $Q / \bar{A} \cong T(R, \mathscr{P}) /(\bar{A} /(\operatorname{Ker} \varphi)$ is an extension of $R$ which has all the aforementioned properties of $T(R, \mathscr{P})$, and, moreover, any ideal of $Q / \bar{A}$ intersects $R$ (viewed as a subring) nontrivially.

REMARK 2. Suppose that $R$ has an involution $\left(^{*}\right)$. Then, for any prime $P$ of degree $j$, there is a prime $P^{*}$ of degree $j$ and an isomorphism $R / P \rightarrow R / P^{*}$ given by $r+P \rightarrow r^{*}+P^{*}$. This isomorphism extends to the algebra of central quotients, and one can check that in the definition of $T(R)$, an involution is induced in Q. Moreover, $V$ is stable under this involution, so $T(R)$ inherits an involution which coincides with $\left(^{*}\right)$ on $R$. Hence the embedding $R \rightarrow T(R)$ is actually an embedding in the category of rings with involution.

\section{REFERENCES}

1. S. A. Amitsur, Prime rings having polynomial identities with arbitrary coefficients, Proc. London Math. Soc., (3), 17 (1967), 470-486.

2. M. Artin, On Azumaya algebras and finite dimensional representations of rings, J. Algebra, 11 (1969), 532-563.

3. J. W. Fisher, Structure of semiprime P.I.-rings, Proc. Amer. Math. Soc., 39 (1973), 465-467.

4. - Structure of semiprime P.I.-rings, II, 1971, (unpublished).

5. E. Formanek, Central polynomials for matrix rings, J. Algebra, 23 (1972), 129132.

6. Frayne, Morel, and Scott, Reduced direct products, Fund. Math., 51, $214 \mathrm{ff}$.

7. A. W. Goldie, A note on prime rings with polynomial identities, J. London Math. Soc., (2), vol. 1 (1969), 606-608.

8. I. N. Herstein, and L. W. Small, Regular elements in P.I.-rings, Pacific J. Math., 36 (1971), 327-330.

9. W. S. Martindale, III, On semiprime P.I.-rings, Proc. Amer. Math. Soc., 40 (1973), 364-369.

10. C. Procesi, On a theorem of M. Artin, J. Algebra, 22 (1972), 309-316.

11. L. Rowen, Some results on the center of a ring with polynomial identity, Bull Amer. Math. Soc., 79 (1973), 219-222.

12. - On rings with central polynomials, J. Algebra, (to appear).

Received July 3, 1973. Research of the first author supported in part by NSF contract GP-38770. 



\section{PACIFIC JOURNAL OF MATHEMATICS}

EDITORS

RICHARD ARens (Managing Editor)

University of California

Los Angeles, California 90024

\section{R. A. Beaumont}

University of Washington

Seattle. Washington 98105

\section{J. DugundjI}

Department of Mathematics University of Southern California Los Angeles, California 90007

D. Gilbarg and J. Milgram

Stanford University

Stanford. California 94305

\section{ASSOCIATE EDITORS}
E. F. BECKENBACH
B. H. NEUMANN
F. WOLF
K. Yoshida

\section{SUPPORTING INSTITUTIONS}

\author{
UNIVERSITY OF BRITISH COLUMBIA \\ CALIFORNIA INSTITUTE OF TECHNOLOGY \\ UNIVERSITY OF CALIFORNIA \\ MONTANA STATE UNIVERSITY \\ UNIVERSITY OF NEVADA \\ NEW MEXICO STATE UNIVERSITY \\ OREGON STATE UNIVERSITY \\ UNIVERSITY OF OREGON \\ OSAKA UNIVERSITY
}

\author{
UNIVERSITY OF SOUTHERN CALIFORNIA \\ STANFORD UNIVERSITY \\ UNIVERSITY OF TOKYO \\ UNIVERSITY OF UTAH \\ WASHINGTON STATE UNIVERSITY \\ UNIVERSITY OF WASHINGTON

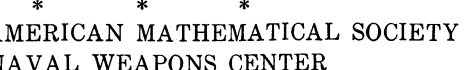

The Supporting Institutions listed above contribute to the cost of publication of this Journal, but they are not owners or publishers and have no responsibility for its content or policies.

Mathematical papers intended for publication in the Pacific Journal of Mathematics should be in typed form or offset-reproduced, (not dittoed), double spaced with large margins. Underline Greek letters in red, German in green, and script in blue. The first paragraph or two must be capable of being used separately as a synopsis of the entire paper. Items of the bibliography should not be cited there unless absolutely necessary, in which case they must be identified by author and Journal, rather than by item number. Manuscripts, in duplicate if possible, may be sent to any one of the four editors. Please classify according to the scheme of Math. Rev. Index to Vol. 39. All other communications to the editors should be addressed to the managing editor, or Elaine Barth, University of California, Los Angeles, California, 90024.

100 reprints are provided free for each article, only if page charges have been substantially paid Additional copies may be obtained at cost in multiples of 50 .

The Pacific of Journal Mathematics is issued monthly as of January 1966. Regular subscription rate: $\$ 72.00$ a year (6 Vols., 12 issues). Special rate: $\$ 36.00$ a year to individual members of supporting institutions.

Subscriptions, orders for back numbers, and changes of address should be sent to Pacific Journal of Mathematics, 103 Highland Boulevard, Berkeley, California, 94708.

\section{PUBLISHED BY PACIFIC JOURNAL OF MATHEMATICS, A NON-PROFIT CORPORATION}

Printed at Kokusai Bunken Insatsusha (International Academic Printing Co., Ltd.), 270, 3-chome Totsuka-cho. Shinjuku-ku, Tokyo 160. Japan.

Copyright (C) 1973 by Pacific Journal of Mathematics Manufactured and first issued in Japan 


\section{Pacific Journal of Mathematics}

\section{Vol. 52, No. $2 \quad$ February, 1974}

Harm Bart, Spectral properties of locally holomorphic vector-valued functions .....

J. Adrian (John) Bondy and Robert Louis Hemminger, Reconstructing infinite

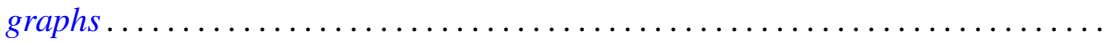

Bryan Edmund Cain and Richard J. Tondra, Biholomorphic approximation of planar

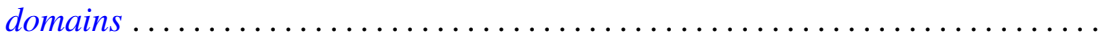

Richard Carey and Joel David Pincus, Eigenvalues of seminormal operators,

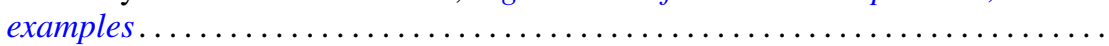

Tyrone Duncan, Absolute continuity for abstract Wiener spaces . . . . . . . . . . . . Joe Wayne Fisher and Louis Halle Rowen, An embedding of semiprime

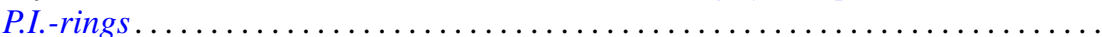

Andrew S. Geue, Precompact and collectively semi-precompact sets of semi-precompact continuous linear operators. . . . . . . . . . . . . . . .

Charles Lemuel Hagopian, Locally homeomorphic $\lambda$ connected plane continua ..... . Darald Joe Hartfiel, A study of convex sets of stochastic matrices induced by

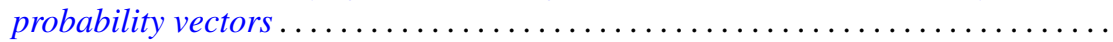

Yasunori Ishibashi, Some remarks on high order derivations $\ldots \ldots \ldots \ldots \ldots \ldots \ldots$ Donald Gordon James, Orthogonal groups of dyadic unimodular quadratic forms.

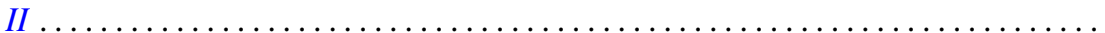

Geoffrey Thomas Jones, Projective pseudo-complemented semilattices . . . . . . . . . Darrell Conley Kent, Kelly Denis McKennon, G. Richardson and M. Schroder,

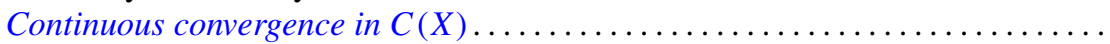

J. J. Koliha, Some convergence theorems in Banach algebras ...

Tsang Hai Kuo, Projections in the spaces of bounded linear oper

George Berry Leeman, Jr., A local estimate for typically real functions . .

475

Andrew Guy Markoe, A characterization of normal analytic spaces by the

homological codimension of the structure sheaf .........

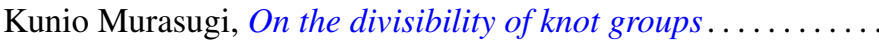

John Phillips, Perturbations of type I von Neumann algebras.

Billy E. Rhoades, Commutants of some quasi-Hausdorff matrices . .

David W. Roeder, Category theory applied to Pontryagin duality

Maxwell Alexander Rosenlicht, The nonminimality of the differential closure .

Peter Michael Rosenthal, On an inversion theorem for the general Mehler-Fock transform pair.

Alan Saleski, Stopping times for Bernoulli automorphisms

John Herman Scheuneman, Fundamental groups of compact complete locally affine complex surfaces. II. ........................

Vashishtha Narayan Singh, Reproducing kernels and operators with a cyclic vector. I. .

Peggy Strait, On the maximum and minimum of partial sums of random variables.

J. L. Brenner, Maximal ideals in the near ring of polynomials modulo 2 .

Ernst Gabor Straus, Remark on the preceding paper: "Ideals in near rings of polynomials over a field" ..........................

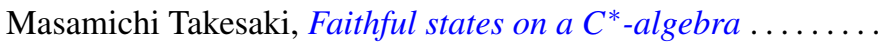

R. Michael Tanner, Some content maximizing properties of the regular simplex.

Andrew Bao-hwa Wang, An analogue of the Paley-Wiener theorem for certain

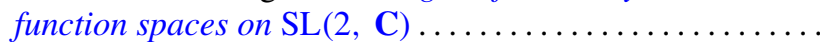

